\title{
PROCEDIMIENTO PARA EL EQUILIBRADO CONTINUO DE OBRAS Y CONSTRUCCIONES
}

\section{(A PROCEDURE FOR WORKS AND BUILDING CONTINUOUS BALANCEMENT)}

\author{
Iñaki Ereño Belaustegui \\ Ingeniería del Suelo Ereño, S. A. \\ Elcano, 11 - 48011 Bilbao \\ Jesús Sáiz de Omeñaca \\ Departamento de Ciencias e Ingeniería de la Tierra, el Terreno y los Materiales, Universidad de Cantabria \\ Avda. de Los Castros, s/n - 39005 Santander
}

ESPAÑA

$400-25$

Fecha de recepción: 16-III-92

\section{RESUMEN}

Se describe un nuevo procedimiento, que permite tanto equilibrar continuamente la parte construida de una obra a medida que se ejecuta como hacerlo posteriormente, aun cuando haya sido entregada y se encuentre en plena utilización.

Este procedimiento consiste básicamente en pretensar el cimiento que va a soportar la carga de la estructura y en mantener a la carga de cálculo las que reciba cada apoyo, independientemente de los cambios que sufran el terreno o el reparto de cargas en el edificio. Para ello, se han diseñado los medios oportunos, que se exponen sucintamente.
SUMMARY

This new procedure allows not only balancing the portion of a work as it is built but also doing this operation once the work has been finished and it is in full use.

Basically, the procedure consists of pre-tautening the foundations on which the load of the structure is going to be supported and keeping added to the reckoned load the ones received by each support without bearing in mind either any ground alteration or the load distribution within the building. In order to achieve this, we have taken the necessary steps, a summary of which is shown below.

\section{INTRODUCCIÓN}

Los edificios y construcciones de diverso tipo se conciben, diseñan y calculan para sí mismos y en función del terreno sobre el que han de asentarse. No obstante, ya durante su ejecución, cualquier obra sufre permanentemente variaciones de carga que afectan a todo tipo de vectores de cálculo y que sobrecargan unos elementos mientras que otros quedan sin carga o reciben esfuerzos de resultante distinta a lo previsto, modificándose el equilibrio de la parte construida mientras se ejecuta la restante.

Una vez finalizada la obra, es corriente que se alteren las previsiones de carga y equilibrio al añadir cargas adicionales, incluso simplemente a causa de un reparto de mobiliario o de personal no previstos.

También son numerosos los cambios que dan lugar a alteraciones del suelo o del subsuelo que pueden afectar a una obra: la excavación o la edificación en un solar contiguo, la realización de un túnel u otras obras en el terreno, las variaciones de la superficie piezométrica o el arrastre de finos por aguas freáticas, etc.

Por fortuna, muchas de estas variaciones pueden ser soportadas y se traducen simplemente en la aparición de pequeños daños, siendo las grietas los más visibles y comunes. Otras veces, aquellos "pequeños daños" son preludio de otros mayores y es preciso intervenir con más o menos urgencia y realizar reparaciones, refuerzos o apuntalamientos con el fin de evitar que toda la construcción resulte afectada.

Como muestra sobradamente la experiencia, el sobredimensionado de las obras no garantiza la robustez de las mismas, sino su encarecimiento y su mayor complejidad e incluso puede ser motivo de deterioro. Efectivamente, en la actualidad, la estructura que soporta los esfuerzos en una obra de ingeniería es monobloque; todos y cada uno de los elementos que la componen son activos dentro del cálculo complejo de equilibrio de esfuerzos. El que una estructura sea robusta no reside precisamente en la sobredimensión. 
A pesar del avance de los conocimientos en este campo y en el de los materiales, la fiabilidad de una construcción, tanto de un edificio como de cualquier obra en general, se confía todavía a la inmovilidad de lo construido y a la inalterabilidad del subsuelo y del entorno. No es extraño que si éstos se alteran (como sucede en la actualidad con frecuencia a causa del intenso aprovechamiento del espacio en las áreas urbano industriales) las obras se degraden, tengan que ser derruidas o incluso que se produzcan catástrofes. Para evitarlo sería preciso incluir en el proyecto medios para controlar el equilibrio de esfuerzos y para restituirlo si se viera alterado por una causa externa.

\section{DESCRIPCIÓN DEL PROCEDIMIENTO}

El procedimiento objeto de este artículo, idea original de I. Ereño, permite tanto equilibrar continuamente la parte construida a medida que se ejecuta la obra como hacerlo posteriormente, aún cuando haya sido entregada y se encuentre en plena utilización. Consiste básicamente en pretensar el cimiento que va a soportar la carga de la estructura y en mantenerlo a la carga de cálculo. Es decir, a medida que varía el peso, se mantiene constante el esfuerzo sobre cada punto o zona de carga descargando o restituyendo la tensión. Sobre esta idea se ha venido trabajando desde 1983, con el fin de desarrollar los medios para la puesta en práctica de la misma.

Esto se consigue interponiendo entre cada pie de carga (muro, columna, pilar o cualquier otra base resistente) y la cabeza del cimiento en que se apoya (sea zapata, pilote, solera, muro o cualquier otra base de apoyo) unos medios para el control dinamométrico de dicha carga y para la elevación o descenso del pie con respecto al cimiento en que se apoya (fig. 1). A través de éste se disponen, con capacidad de deslizamiento axil, unos tirantes tensores, con su correspondiente bulbo terminal. Hemos desarrollado también un mecanismo que permite que de aquel bulbo partan a su vez dos o más bifurcaciones con sus extremos firmemente anclados en el terreno.

Tal y como puede comprenderse con la ayuda de la fig. 1, la cabeza del cimiento (2), con su cápsula (7), juega a modo de pistón en la cámara que prolonga al pie (6). Puede, además, bascular mediante un juego de rótula variando el plano de asentamiento del fundamento, sin sobrepasar el coeficiente de esfuerzo cortante del terreno, para que el conjunto se beneficie siempre del máximo apoyo. Por otra parte, los elementos de regulación (10), actuando a la vez sobre el ala (9) y sobre la base (8) permiten ajustar la altura. Existe siempre un apoyo total gracias a la acción compensadora de los elementos de elevación y/o descenso.

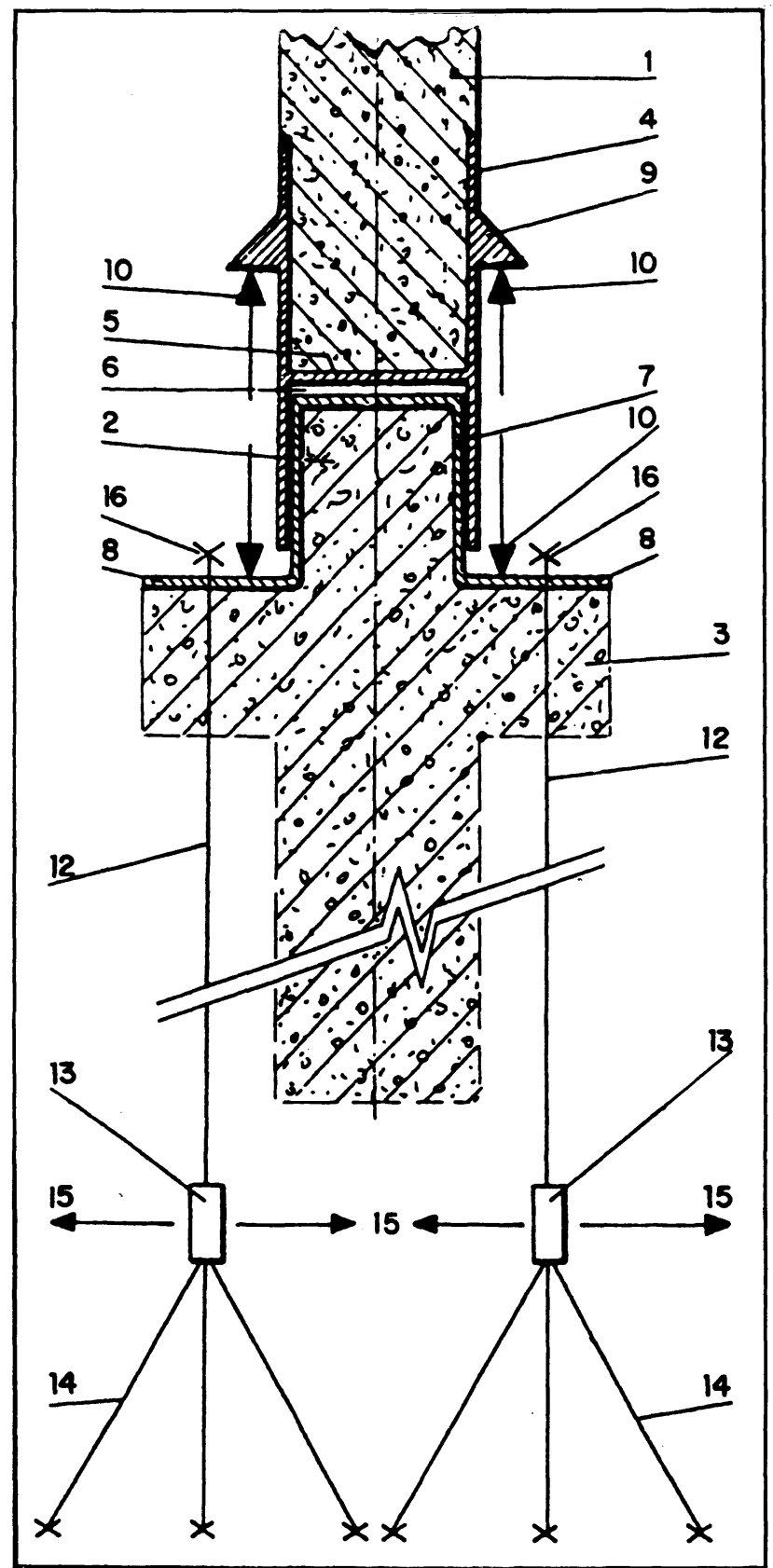

Fig. 1.-Sección esquemática de un apoyo y de su fundación. Se han representado también los tensores y sus bulbos y ramificaciones.

1.-Pie del apoyo. 2.-Cabeza del cimiento. 3.-Fundamento 4.-Collar del pie. 5.-Tabique diametral. 6.-Cámara. 7.-Cápsula que recubre la cabeza del cimiento. 8.-Base de la cápsula, que recubre al fundamento y en la que existen orificios de paso para los tirantes. 9.-Ala resistente del collar. 10.-Elementos de regulación de altura (p. ej., gatos hidráulicos o husillos de vis sin fin), simbolizados por flechas. 12.-Tirantes tensores. 13.-Bulbos. 14.-Ramas de los tirantes. 15.-Componentes de tiro perpendiculares a las isobaras del bulbo. 16.-Cabeza tensora de los tirantes. 


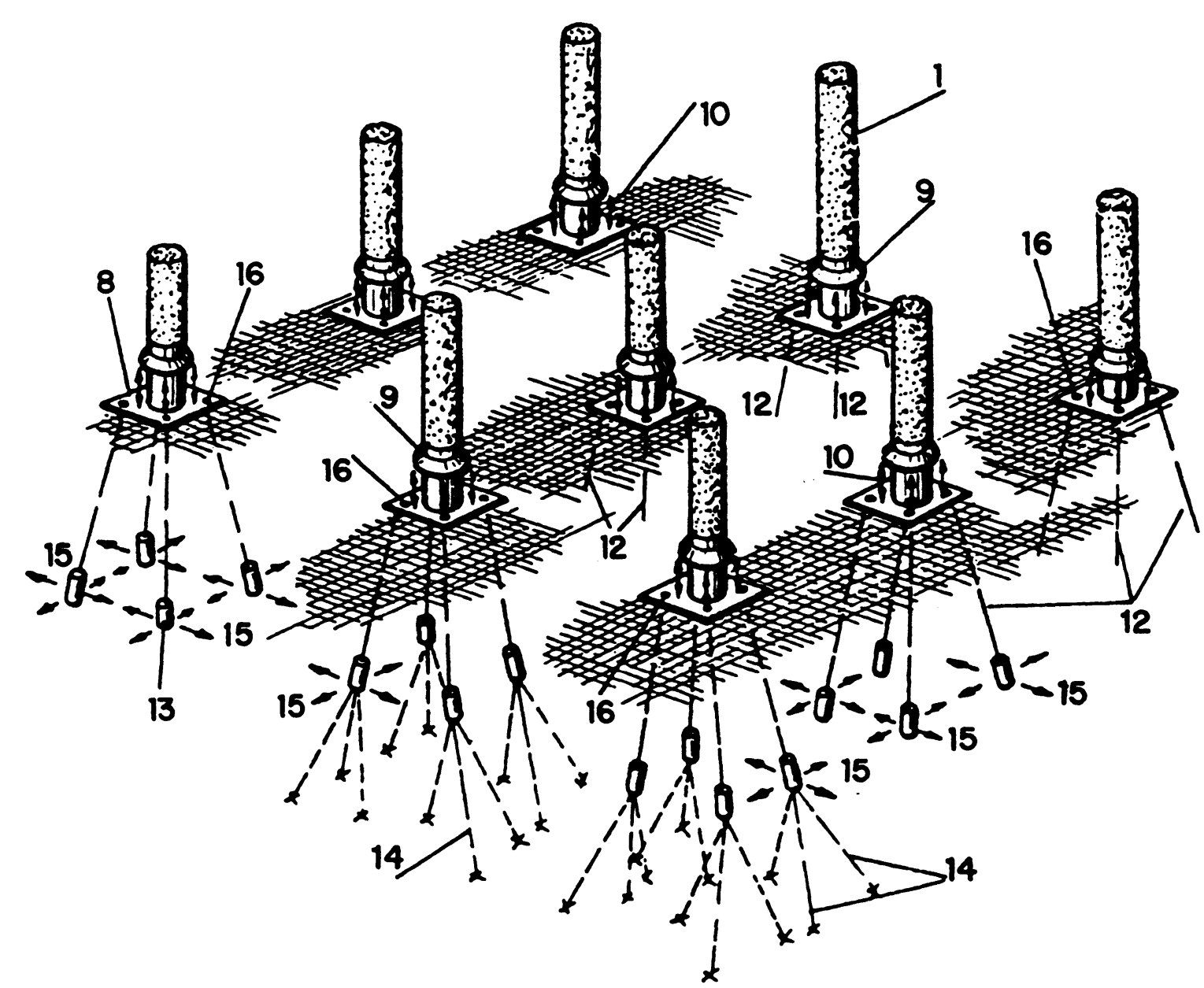

Fig. 2.-Representación de un conjunto de apoyos. La resultante de los componentes de tiro del esfuerzo de tracción determina una presión radial que compacta el terreno y eleva su resistencia.

1.-Pie del apoyo. 8.-Base de la cápsula, que recubre a la zapata. 9.-Ala resistente del collar. 10.-Elementos de regulación de altura, simbolizados por flechas. 12.-Tirantes tensores. 13.-Bulbos. 14.-Ramas de los tirantes. 15.-Componentes de tiro perpendiculares a las isobaras del bulbo. 16.-Cabeza tensora de los tirantes.

La tracción ejercida por la cabeza tensora (que en los ensayos y aplicaciones realizados fue un mecanismo hidráulico) de cada tirante se transmite al bulbo y a las ramas y da lugar a vectores sensiblemente perpendiculares a los isobaras del bulbo. Al operar un conjunto de tirantes (fig. 2), la presión radial ejercida por cada bulbo entra en contraposición con la que ejercen los adyacentes, por lo que dan lugar colectivamente a una compactación del terreno en el contexto de los apoyos de carga, incrementando favorablemente el ángulo de rozamiento interno y otros parámetros. El equilibrio de esfuerzos en el conjunto de un edificio u obra puede mantenerse al ser posible controlar y operar selectivamente sobre los distintos tensores.

Puede decirse que no existen antecedentes a este método, e incluso los que citamos como relativamente próximos $(1,2)$ tienen objetivos distintos y siguen procedimientos que difieren en lo fundamental y en los detalles. Por otra parte, podría pensarse que ciertos mecanismos que denuncian sobreesfuerzos en partes de- terminadas de una obra lo son, pero en realidad no permiten por sí mismos controlar y restablecer el equilibrio de esfuerzos. Citemos, por ejemplo, las plomadas previstas en las presas hidráulicas que permiten determinar cuándo existe una sobrepresión en el muro; no es operando sobre el mecanismo, que sólo actúa como testigo, como se restablece la presión de cálculo, sino evacuando agua para disminuir el esfuerzo soportado.

\section{FUNCIONAMIENTO DEL SISTEMA}

Inicialmente, todos los tirantes se someten a las tensiones de cálculo, de manera que ya al inicio de la obra se maximiza la resistencia del terreno y el equilibrio de fuerzas se carga en los fundamentos. A medida que progresa la obra, los pies van ejerciendo una carga añadida, por lo que se van aflojando los tensores o tirantes para mantener las tensiones de cálculo hasta concluir la construcción. Entonces, la totalidad de la obra debe quedar en equilibrio.

http://informesdelaconstruccion.revistas.csic.es 
Una pérdida de esfuerzo de carga en cualquiera de las zapatas significa que ésta ha cedido por cualquier efecto no deseable. Basta tensar los tirantes para restablecer la carga y actuar sobre los elevadores para equilibrar la carga de trabajo del pie y restablecer el equilibrio de esfuerzos de todo el conjunto.

También es posible situar en cada unidad generadora de fuerza un sensor extensiométrico, que conectado a circuitos de transducción envíe señales digitalizadas a una Unidad Central de Procesado de Datos. Podrian así tanto detectarse las anomalías como mantenerse permanentemente los parámetros que determinen el equilibrio de la obra, bien manualmente, bien de forma automática mediante un programa informático adecuado. No es necesario extenderse sobre las ventajas del sistema para evitar emergencias.

Si en una obra dotada del sistema que se presenta se detectara repetidamente la necesidad de corregir presiones o esfuerzos en una parte determinada, se podría deducir de forma rápida y segura donde se está produciendo el fenómeno indeseable. Muy probable- mente, su origen y naturaleza podrán conocerse por medios sencillos (quizá una simple inspección ocular de obras cercanas, quizá unos sondeos en lugares ya predeterminados) en vez de tener que acudir a calicatas y sondeos apresurados, casi al azar y seguramente mucho más onerosos. En todo caso, en vez de producirse daños más o menos graves, la edificación podrá mantenerse en equilibrio, sin grietas y con absoluta fiabilidad.

\section{EPILOGO}

Aunque estamos convencidos de la bondad del procedimiento, somos conscientes de su relativa complejidad, de que su aplicación no es sencilla y de que es muy conveniente su desarrollo ulterior. Esperamos que la patente del procedimiento, recientemente obtenida, permita no sólo evaluar pública y adecuadamente los ensayos y aplicaciones realizados hasta el momento, sino también efectuar y valorar otros nuevos con participación de diversos profesionales de reconocida solvencia.

\section{BIBLIOGRAFÍA}

(1) Freyssinet, E. Procédé et dispositifs de réalisation de fondations d'ouvrages maritimes. Brevet d'invention Gr. 7 - cl. 2, núm. 1.063.854. Ministèré de L'Industrie et du Commerce, Service de la Propièté Industrielle, 4 pp., 1 planche. París, 1954.

(2) Terrapin Ltd. Pieds ou empattements pour supporter des constructions. Brevet d'invention PV núm. 909.216, núm. 1.333.455. Ministèré de L'Industrie et du Commerce, Service de la Propièté Industrielle, 3 pp., 4 planches. París, 1963.

\section{publicación del ICCET/CSIC}

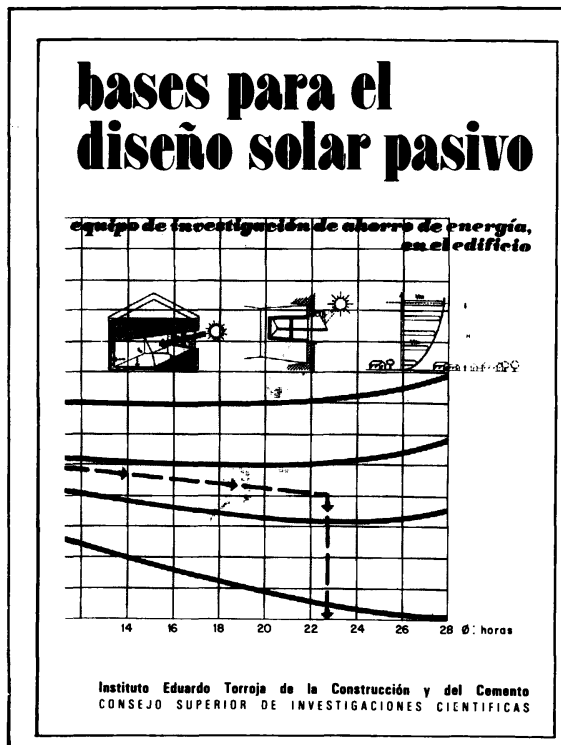

Equipo de Ahorro de Energía en el edificio

Dirección y coordinación: Arturo García Arroyo

M. a José Escorihuela

José Luis Esteban

José Miguel Frutos

Manuel Olaya

Bernardo Torroja
Las dificultades de suministro y el alto coste de los productos energéticos convencionales han despertado la atención de los usuarios, técnicos $\mathrm{e}$ industriales de la edificación hacia los procedimientos y sistemas en que se basa el aprovechamiento de otras fuentes alternativas de energía, principalmente la solar. Esto ha generado un rápido desarrollo industrial y comercial que, en opinión de los autores de este libro, arrastran los siguientes defectos: un mimético tecnologismo respecto de los sistemas convencionales que violenta las pe culiaridades de la energia solar (baja densidad y variabilidad en el tiempo), y una escasa selectividad en la aplicación de los sistemas y procedimientos pasivos dando origen a un ecumenismo arquitectónico solar, al margen de las condiciones climáticas y funcionales específicas de cada caso y lugar.

En este libro, utilizando criterios y metodologia pedagógicos, se dan los fundamentos e instrumentos teórico-prácticos necesarios para el planteamiento de todo proyecto arquitectónico solar pasivo, de acuerdo con los principios éticos y económicos de conservación y ahorro de energia. Es decir: respeto de los presupuestos bioclimáticos, búsqueda de la máxima captación y acumulación de la radiación solar, y esmero en el aislamiento térmico de los cerramientos.

Un volumen encuadernado en cartulina ibiza plastificada, a cinco colores, de $16 \times 23 \mathrm{~cm}$, compuesto de 216 páginas, 217 figuras, 87 gráficos, 19 tablas y 10 cuadros. 